\title{
EFFECT OF SUBSTRATE HEATING ON PERPENDICULAR ANISOTROPY IN Co/Pt MULTILAYERED FILMS
}

\author{
S. SHIOMI, T. MIYAUCHI, M. MASUDA and S. YAHAGI*
}

Department of Electronics, Mie University, 1515 Kamihama-cho, Tsu 514, Japan

*New Materials Research Lab., Daido Steel Co., Ltd., 2-30 Daido-cho, Minami-ku, Nagoya 457, Japan

\begin{abstract}
Co/Pt multilayered films were prepared on heated glass slides by electron beam evaporation. It was found that perpendicular anisotropy $K_{1}$ increased with elevating substrate temperature $T_{s}$. Interface anisotropy $K_{s}$ and volume anisotropy $K_{v}$ were evaluated from the dependence of $K_{1}$ on bilayer period $\lambda$. In the range of $T_{\mathrm{s}}$ from 30 to $130^{\circ} \mathrm{C}, K_{\mathrm{s}}$ increased with elevating $T_{\mathrm{s}}$ while $K_{\mathrm{v}}$ hardly depended on $T_{\mathrm{s}}$. On the other hand, at $T_{s}=300^{\circ} \mathrm{C}, K_{s}$ was almost zero but $K_{v}$ was positive and large, which made $K_{\perp}$ almost independent of $\lambda$. It was also found that substrate heating was quite effective to enhance coercive force $H_{c_{1}}$. Though $K_{1}$ was hardly dependent on Pt buffer layer thickness, $H_{c 1}$ increased with thickening Pt buffer layer. There was no correlation between low angle X-ray diffraction intensity $I_{1}$ and $K_{s}$ or between $I_{1}$ and $K_{l}$. Though the dispersion of (111) orientation, $\Delta \theta_{50}$, decreased with elevating $T_{s}$, there was no clear correlation between $\Delta \theta_{50}$ and $K_{1}$ or between $\Delta \theta_{50}$ and $H_{\mathrm{c}}$. The films deposited on room temperature substrates were annealed in vacuum. With elevating annealing temperature $T_{a}, K_{\mathrm{s}}$ decreased while $K_{v}$ slightly increased. Though $\Delta \theta_{50}$ was hardly changed by annealing, $I_{1}$ decreased with elevating $T_{a}$.
\end{abstract}

KEY WORDS:Co/Pt MULTILAYERED FILM, VACUUM EVAPORATION, SUBSTRATE HEATING, MAGNETIC ANISOTROPY, COERCIVE FORCE, ANNEALING, X-RAY DIFFRACTION

\section{INTRODUCTION}

Recent investigations have revealed that Co/Pt multilayered films have large perpendicular anisotropy [1] as well as the enhanced magneto-optical Kerr rotation at shorter wavelengths [2]. Co/Pt multilayered films are consequently regarded as one of the most promising candidates for future high-density magneto-optical recording media.

Perpendicular anisotropy in Co/Pt multilayered films has been attributed to interface anisotropy[2]. It is well known that an increase in the substrate temperature increases the surface mobility of the condensed atoms [3], which would cause the smoothing of layered structure. On the other hand, interdiffusion between adjacent layers increases with elevating substrate temperature, which would affect not only interface anisotropy but also volume anisotropy through the formation of CoPt alloy layers. Accordingly, it is quite interesting to investigate the effect of substrate heating on perpendicular anisotropy in multilayered films.

In this paper we report the effects of substrate heating, Pt buffer layer thickness and annealing in vacuum on magnetic properties of evaporated Co/Pt multilayered films.

\section{EXPERIMENTAL}

Co and Pt layers were alternately deposited by electron beam evaporation on to glass slides heated up to $300^{\circ} \mathrm{C}$. The layer thickness was monitored using quartz monitors. The thickness ratio of Co layer to Pt layer is kept constant at $3: 10$. Though the bilayer period $\lambda$ was varied from 1.3 to $6.5 \mathrm{~nm}$, the total film thickness $d$ was kept constant around $40 \mathrm{~nm}$ by adjusting the number of bilayers. The deposition was started from and ended with Pt layers in any film. In some films, the Pt layer deposited directly onto a substrate was thickened to $15 \mathrm{~nm}$ irrespective of $\lambda$ in order to investigate the effect of buffer layer. The deposition rate was $0.4 \mathrm{~nm} / \mathrm{min}$ for both Co and Pt layers. The pressure during deposition was $5 \times 10^{-6}$ Torr.

Magnetic properties were measured using a vibrating sample magnetometer and a torque magnetometer. The effective perpendicular anisotropy constant $K_{l}$ was calculated for the total film volume except that of a $15 \mathrm{~nm}$ thick Pt buffer layer. Crystalline properties were investigated by X-ray diffraction (XRD). The bilayer period $\lambda$ obtained by low angle XRD reasonably agreed with the designed value. The bilayer periods given below are designed ones. 


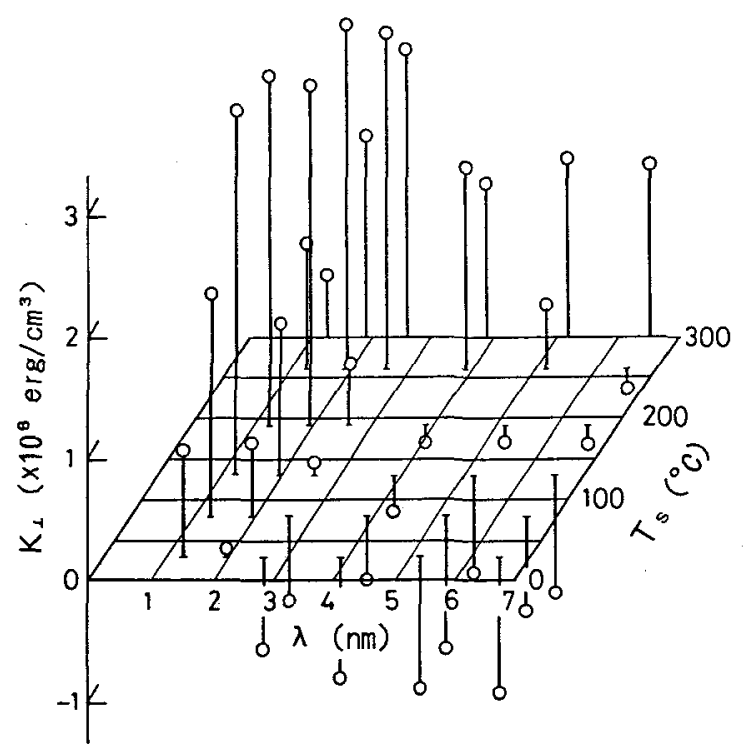

Fig.1 Perpendicular anisotropy $K_{1}$ as a function of bilayer period $\lambda$ and substrate temperature $T_{\mathrm{s}}$.

\section{RESULTS AND DISCUSSION}

Figure 1 shows perpendicular anisotropy $K_{1}$ as a function of bilayer period $\lambda$ and substrate temperature $T_{\mathrm{s}}$. Without substrate heating or at $T_{\mathrm{s}}=30^{\circ} \mathrm{C}, K_{1}$ decreases with increasing $\lambda$, being negative in films with $\lambda \geq 2.6 \mathrm{~nm}$. As $T_{\mathrm{s}}$ is elevated, however, $K_{\perp}$ becomes larger. In films with $\lambda=1.3 \mathrm{~nm}$, $K_{\perp}$ reaches the maximum of $3 \times 10^{6} \mathrm{erg} / \mathrm{cm}^{3}$ at $T_{\mathrm{s}}=130^{\circ} \mathrm{C}$, which is three times larger than that at $T_{\mathrm{s}}=30^{\circ} \mathrm{C}$. In films prepared at lower $T_{\mathrm{s}}, K_{1}$ decreases with increasing $\lambda$, which suggests that $K_{1}$ is mainly due to interface anisotropy. In films prepared at $T_{\mathrm{s}}=300^{\circ} \mathrm{C}$, however, $K_{\perp}$ never decreases with increasing $\lambda$, which suggests that $K_{1}$ is due to volume anisotropy rather than interface anisotropy.

Figure 2 shows the product of bilayer period $\lambda$ and perpendicular anisotropy $K_{1}$, $\lambda \times K_{1}$, in films prepared at different substrate temperatures as a function of co layer thickness $t_{\text {co }}$. At lower $T_{\text {s }}$ up to 130 ${ }^{\circ} \mathrm{C}, \lambda \times K_{\perp}$ decreases almost linearly with increasing $t_{c_{0}}$, as reported previously [2]. The interface anisotropy $K_{\mathrm{s}}$ and volume anisotropy $K_{v}$ were evaluated from the relation $\lambda \times K_{\perp}=K_{v} t_{c_{0}}+2 K_{s}$. As shown in Tab.1, $K_{s}$ increases with elevating $T_{s}$, which suggests that interface becomes smoother

Tab.1 Interface anisotropy $K_{\mathrm{s}}$ and volume anisotropy $K_{v}$ at substrate temperature $T_{\mathrm{s}}$.

\begin{tabular}{ccc}
\hline$T_{\mathrm{s}}\left({ }^{\circ} \mathrm{C}\right)$ & $K_{\mathrm{s}}\left(\mathrm{erg} / \mathrm{cm}^{2}\right)$ & $K_{\mathrm{V}}\left(\mathrm{erg} / \mathrm{cm}^{3}\right)$ \\
\hline 30 & 0.15 & $-7.1 \times 10^{6}$ \\
80 & 0.18 & $-6.5 \times 10^{6}$ \\
130 & 0.30 & $-8.0 \times 10^{6}$ \\
300 & 0.01 & $+6.1 \times 10^{6}$ \\
\hline
\end{tabular}

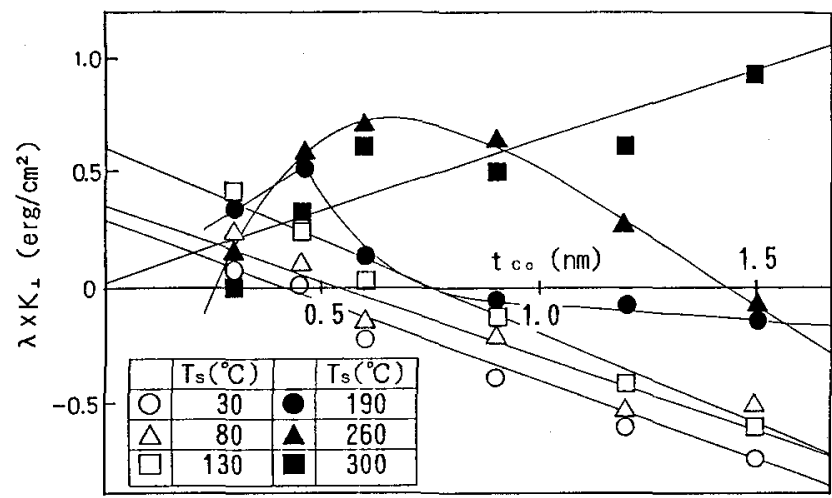

Fig.2 Bilayer period $\lambda$ times anisotropy $K_{\mathfrak{L}}$ vs Co layer thickness $t_{\mathrm{c}}$.

and sharper with elevating $T_{s}$. On the other hand, $K_{v}$ hardly depends on $T_{\mathrm{s}}$, which suggests that interdiffusion between Co and Pt layers remains insignificant in this temperature range. The similar increase in $K_{\mathrm{s}}$ with elevating $T_{\mathrm{s}}$ has been reported for Co/Pd multilayered films [4]. Though there seems no linear relation between $\lambda \times K_{\perp}$ and $t_{\mathrm{co}}$ at $T_{\mathrm{s}}=190$ and $260^{\circ} \mathrm{C}$, $\lambda \times K_{1}$ increases almost linearly with increasing $t_{\text {co }}$ at $T_{\mathrm{s}}=300^{\circ}$. As shown in Tab.1, $K_{s}$ is nearly zero, which suggests that Copt alloy layers are formed at interfaces by interdiffusion. On the other hand, $K$. is positive and as large as $6.1 \times 10^{6} \mathrm{erg} /$ $\mathrm{cm}^{3}$. This volume anisotropy might be related to the perpendicular anisotropy observed in sputtered CoPt alloy films $[5,6]$.

Figure 3 shows coercive force $H_{c_{1}}$ as a function of bilayer period $\lambda$ and substrate temperature $T_{s}$, where $H_{c_{1}}$ was determined from the magnetization curve with applied field normal to the film plane.

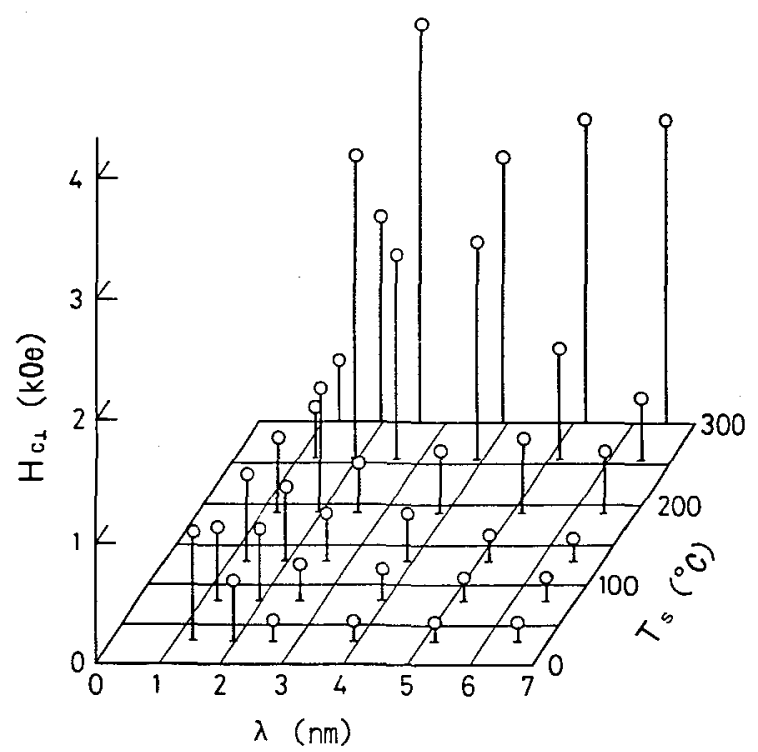

Fig. 3 Coercive force $H_{c_{1}}$ as a function of bilayer pericx $\lambda$ and substrate temperature $T_{\mathrm{s}}$. 


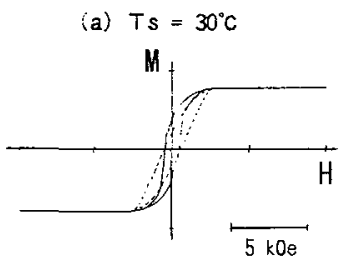

(b) $\mathrm{Ts}=80^{\circ} \mathrm{C}$

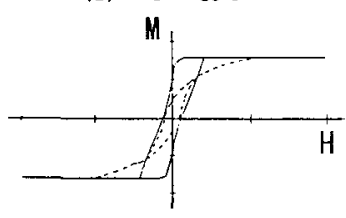

(c) $T s=130^{\circ} \mathrm{C}$

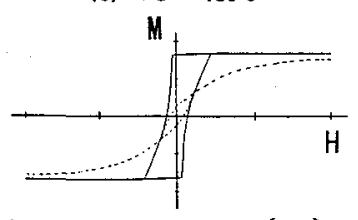

Fig.4 Perpendicular(-) and in-plane(--) magnetization curves in films prepared at different substrate temperatures. Bilayer period is $2.0 \mathrm{~nm}$.

In films prepared at $T_{\mathrm{s}} \leqq 190^{\circ} \mathrm{C} H_{\mathrm{c} \perp}$ is small, never exceeding 1 kOe. By elevating $T_{\mathrm{s}}$ to $260^{\circ} \mathrm{C}$ and higher, however, very high coercive force can be obtained. The highest one was $3.3 \mathrm{kOe}$ in the film with $\lambda=2.6$ $\mathrm{nm}$ prepared at $T_{\mathrm{s}}=300^{\circ} \mathrm{C}$.

The magnetization curves of films prepared at different substrate temperatures are shown in Fig.4, where the bilayer period is constant at $2.0 \mathrm{~nm}$. The solid and dashed curves are the magnetization curves with applied field normal and parallel to the film plane, respectively. The squareness $S_{1}$, which is defined by the ratio of residual to saturation magnetization or $S_{1}=M_{r_{1}} / M_{s}$, increases with elevating $T_{\mathrm{s}}$, reaching unity at $T_{\mathrm{s}}=130^{\circ} \mathrm{C}$. Coercive force $H_{c_{1}}$ also increases with elevating $T_{\mathrm{s}}$, reaching the maximum of $2.5 \mathrm{kOe}$ at $T_{\mathrm{s}}=260^{\circ} \mathrm{C}$. However, at $T_{\mathrm{s}}=300^{\circ} \mathrm{C}$, both $S_{1}$ and $H_{c_{1}}$ decrease to values somewhat smaller than those at $T_{\mathrm{s}}=260^{\circ} \mathrm{C}$.

Low angle $X$-ray diffraction peaks were observed in any film prepared at $T_{\mathrm{s}}$ up to $190^{\circ} \mathrm{C}$. However, at $T_{\mathrm{s}}=260^{\circ} \mathrm{C}$, clear diffraction peaks were observed only in films with $\lambda \geq 2.0 \mathrm{~nm}$. At $T_{\mathrm{s}}=300^{\circ} \mathrm{C}$, clear diffraction peaks were not observed until $\lambda$ was increased to $6.5 \mathrm{~nm}$. This result suggests that layered structure becomes less sharp due to interdiffusion with elevating $T_{\mathrm{s}}$. There was no correlation between low angle XRD intensity $I_{1}$ and $K_{1}$ or between $I_{1}$ and $K_{s}$.

It has been pointed out that films with higher (111) orientation have larger perpendicular anisotropy[7]. We also estimated the degree of (111) orientation by

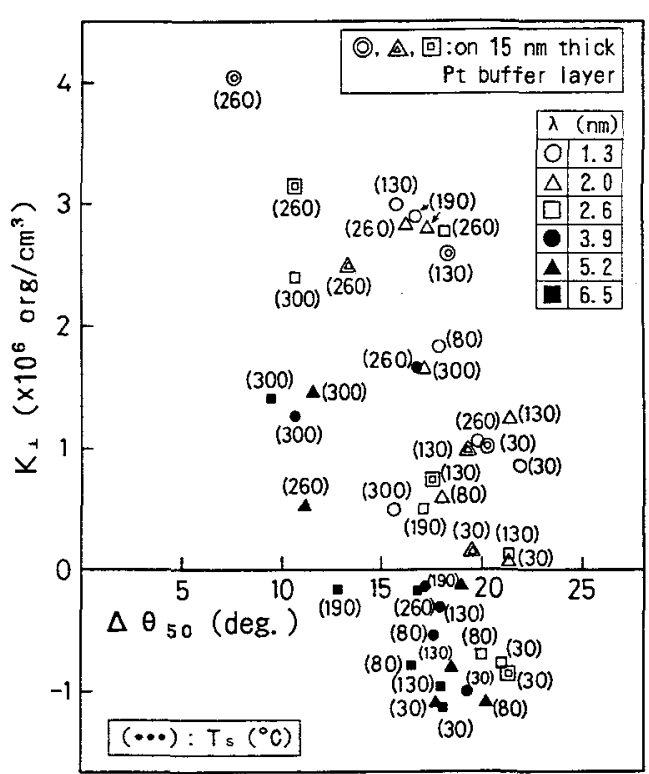

Fig.5 Anisotropy $K_{1}$ vs dispersion of (111) orientation, $\Delta \theta_{50} . T_{\mathrm{s}}$ is shown in parentheses.

measuring rocking curves. Figure 5 shows the relation between $K_{\perp}$ and $\Delta \theta_{50}$, the FWHM of a rocking curve ( $\mathrm{Cu}-\mathrm{K} \alpha$ radiation). Films with different bilayer periods are shown by different symbols with substrate temperatures in parentheses. It is seen that $\Delta \theta_{50}$ tends to decrease with elevating $T_{s}$. However, there is no clear correlation between $K_{1}$ and $\Delta \theta_{50}$. No correlation was found between $H_{c_{1}}$ and $\Delta \theta_{50}$ either. The increase in $H_{c} 1$ with elevating $T_{\mathrm{s}}$ might be caused by the growth of crystallites.

Figure 6 shows the effect of buffer layer thickness on perpendicular anisotropy $K_{1}$ and coercive force $H_{c_{1}}$. The films shown by open circles have $15 \mathrm{~nm}$ thick buffer layers while those shown by closed circles have 1 to $2 \mathrm{~nm}$ thick buffer layers depending on bilayer period. It is seen that $K_{1}$ hardly depends on the thickness of a Pt buffer layer except in a few films. On the other hand, $H_{\mathrm{cl}}$ increases with thickening a $\mathrm{Pt}$ buffer layer in any film. The most remarkable increase in $H_{\mathrm{c} \perp}$ as well as in $K_{\perp}$ is obtained in the film with $\lambda=1.3 \mathrm{~nm}$ prepared at $T_{\mathrm{s}}=260^{\circ} \mathrm{C}$. The similar effect of $P t$ buffer layer on $K_{1}$ and $H_{c_{1}}$ has been reported for sputtered Co/Pt multilayered films [8].

Low angle $X$-ray diffraction intensities hardly depended on buffer layer thickness. However, $\Delta \theta_{50}$ in films prepared at $T_{\mathrm{s}}=260^{\circ} \mathrm{C}$ is much smaller than those at $T_{s}=30$ and $130^{\circ} \mathrm{C}$, as shown in Fig.5. This is due to the fact that the (111) orientation of $15 \mathrm{~nm}$ thick Pt layer deposited at $T_{\mathrm{s}}=260^{\circ} \mathrm{C}$ is much higher than those at $T_{\mathrm{s}}=$ 30 and $130^{\circ} \mathrm{C}$. 


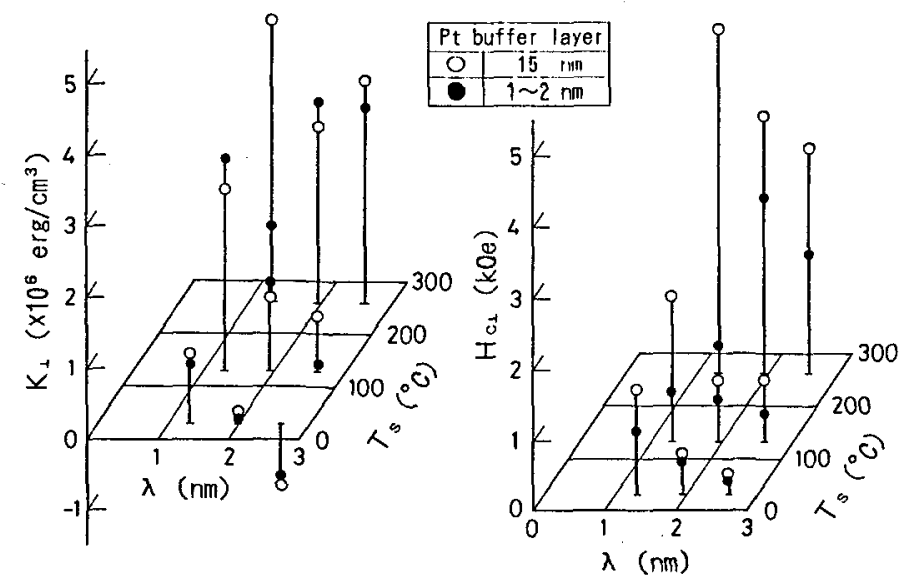

Fig.6 $K_{1}$ and $H_{c_{1}}$ in films with $(\mathrm{O})$ and without (O) $15 \mathrm{~nm}$ thick $\mathrm{Pt}$ buffer layer as a function of $\lambda$ and $T_{\mathrm{s}}$.

We also investigated the effect of annealing on magnetic properties. Films with different bilayer periods prepared on room temperature substrates were annealed in vacuum at the pressure of $3 \times 10^{-7}$ Torr. They were kept at annealing temperature $T_{\mathrm{a}}$ for two hours. Though $K_{1}$ decreased with elevating $T_{a}$ in films with smaller bilayer periods, it hardly depended on $T_{\mathrm{a}}$ or slightly increased with elevating $T_{\mathrm{a}}$ in films with larger bilayer periods. The interface anisotropy $K_{s}$ and volume anisotropy $K_{v}$ were evaluated at each annealing temperature $T_{\mathrm{a}}$ and shown in Tab.2. With elevating $T_{\mathrm{a}}, K_{\mathrm{s}}$ decreases while $K_{\mathrm{v}}$ slightly increases. It was found that low angle XRD diffraction became less intensive as $T_{a}$ became higher. However, $\Delta \theta_{50}$ was hardly affected by annealing. We also found that $H_{\mathrm{c}_{1}}$ decreased with elevating $T_{\mathrm{a}}$ in films with $\lambda=1.3 \mathrm{~nm}$. This might result from the decrease in $K_{1}$ with elevating $T_{\mathrm{a}}$.

\section{CONCLUSIONS}

We prepared Co/Pt multilayered films on heated glass slides by electron beam evaporation. Perpendicular anisotropy $K_{1}$ increased with elevating substrate temperature $T_{\mathrm{s}}$. In the range of $T_{\mathrm{s}}$ from 30 to $130^{\circ} \mathrm{C}$, interface anisotropy $K_{s}$ increased with elevating $T_{\mathrm{s}}$ while volume anisotropy $K_{v}$ hardly depended on $T_{\mathrm{s}}$. On the other hand, at $T_{\mathrm{s}}=300^{\circ} \mathrm{C}, K_{\mathrm{s}}$ was almost zero but $K_{v}$ was positive and large.

We found that substrate heating was quite effective to enhance coercive force $H_{c_{1}}$. Further increase in $H_{c_{1}}$ was obtained by thickening $P t$ buffer layer.

The low angle XRD intensity $I_{1}$ and dispersion of $(111)$ orientation, $\Delta \theta_{50}$, also
Tab.2 Interface anisotropy $K_{\mathrm{s}}$ and volume anisotropy $K_{\mathrm{v}}$ in films annealed at $T_{\mathrm{a}}$.

\begin{tabular}{ccc}
\hline$T_{\mathrm{a}}\left({ }^{\circ} \mathrm{C}\right)$ & $K_{\mathrm{s}}\left(\mathrm{erg} / \mathrm{cm}^{2}\right)$ & $K_{\mathrm{v}}\left(\mathrm{erg} / \mathrm{cm}^{3}\right)$ \\
\hline $30^{*}$ & $0.15^{*}$ & $-7.1 \times 10^{6 *}$ \\
80 & 0.12 & $-6.1 \times 10^{6}$ \\
130 & 0.12 & $-6.0 \times 10^{6}$ \\
190 & 0.10 & $-5.4 \times 10^{6}$ \\
260 & 0.08 & $-5.4 \times 10^{6}$ \\
300 & 0.08 & $-5.3 \times 10^{6}$ \\
\hline${ }^{*}$ As deposited.
\end{tabular}

depended on $T_{\mathrm{s}}$. However, there was no clear correlation between $I_{1}$ and $K_{\perp}$ or between $\Delta \theta_{50}$ and $K_{1}$. No correlation was found between $\Delta \theta_{50}$ and $H_{c_{1}}$ either.

Annealing the films prepared on room temperature substrates in vacuum, we found that $K_{s}$ decreased while $K_{v}$ slightly increased with elevating annealing temperature $T_{\text {a }}$. Though $\Delta \theta_{50}$ was hardly affected by annealing, $I_{1}$ decreased with elevating $T_{\mathrm{a}}$.

Further investigations, especially those on microstructures of films, are required to elucidate why $K_{\perp}$ and $H_{c_{1}}$ are enhanced by substrate heating.

\section{ACKNOWLEDGMENTS}

The authors thank Dr. S. Tsunashima of Nagoya University for his helpful advice. The authors also thank Messrs. K. Maeda, S. Seguchi and T. Nishimura for their help in experiments.

\section{REFERENCES}

[1] P.F.Carcia: J.Appl.Phys., 63, 5066 (1988).

[2] W.B.Zeper, F.J.A.M.Greidanus, P.F. Carcia and C.R.Fincher: J.Appl.Phys., 65 , 4971 (1989).

[3] R.W.Berry, P.M.Hall and M.T.Harris: "Thin Film Technology", Krieger, 1979, p. 130.

[4] F.J.A.den Broeder, W.Hoving and P.J.H. Bloemen: presented at E-MRS'90 Strasbourg Meeting, France.

[5] S.Shiomi, S.Koura, H.Okazawa and M. Masuda: Res.Rep.Fac.Eng.Mie Univ., 15, 45 (1990).

[6] M.Yamasaki, M.Nawate, S.Honda and T. Kusuda: Digests of the 14 th Annual Conference on Magnetics in Japan, Matsuyama, Oct.8-11, 1990, p.367.

[7] M.Hasegawa, K.Nakamura, S.Tsunashima and S.Uchiyama: Digests of the 14 th Annual Conference on Magnetics in Japan, Matsuyama, Oct.8-11, 1990, p.64.

[8] S.Honda, H.Tanimoto and T.Kusuda: IEEE Trans.Magn., 26, 2730 (1990). 